\title{
QUÍMICA MINERAL EM GRANADA DE DIFERENTES NIVEIS CRUSTAIS
}

\author{
Danielle Piuzana (*), Cristiane Castañeda (*), Márcio Martins Pimentel (**), \\ ReinhardtA. Fuck $(* *)$ \& Willian T. Soares $(* * *)$
}

\begin{abstract}
Crystallochemical standards of garnet from rocks in different metamorphic grades generated during the Brasiliano Orogeny were investigated. Four kinds of rocks containing garnet were selected: ultrahigh temperature granulite, granulitized granites, paragranulites and kyanite-garnet-muscovite schist. End-member calculations indicate significant variations in $\mathrm{Fe}$ and $\mathrm{Mg}$ contents, and little variation in $\mathrm{Ca}$ and $\mathrm{Mn}$. All analyzed garnet samples belong to the almandine-pyrope series. Garnet from micaschist presents the highest amount (\%) of Fe molecules $\left(\mathbf{a l m}_{76} \mathbf{p y}_{15} \mathbf{g r o}_{6} \mathbf{s p}_{2}\right)$, followed by garnet of granulitized granites $\left(\mathbf{a l m}_{69-66} \mathbf{p y}_{20-26} \mathbf{g r o}_{6-9} \mathbf{s} \mathbf{p}_{2-3}\right)$, paragranulites $\left(\mathbf{a l m}_{62-52} \mathbf{p y _ { 4 2 - 3 2 }} \mathbf{g r o}_{4-6} \mathbf{s p}_{1-2}\right)$ and UHT granulite $\left(\operatorname{alm}_{63} \mathrm{py}_{23}\right.$ gro $\left._{13} \mathrm{sp}_{1}\right)$.

In all analysed garnet grains rims have higher iron content than cores. Mg and Ca contents in UHT granulite garnet present a negative correlation with Fe, showing gradual decrease of these elements towards the crystal rim. On the other hand, in granulitized granites, $\mathrm{Ca}$ and $\mathrm{Mg}$ remain constant throughout the crystal. Garnet crystals of UHT granulite and granulitized granites, which have the oldest $\mathrm{Sm}-\mathrm{Nd}$ isochron ages (633-625 Ma), display the highest values of $\mathrm{Mg} /(\mathrm{Mg}+\mathrm{Fe})$. Garnet from paragranulite, with Sm-Nd isochron age of $606 \mathrm{Ma}$, presents high values of $\mathrm{Ca} /(\mathrm{Ca}+\mathrm{Mg})$. The dispersion seems to indicate that the isotopic system garnet in samples of paragranulite was opened due to retrometamorphic and/or deformational events.
\end{abstract}

\section{RESUMO}

Padrões cristaloquímicos em granadas, geradas durante a orogenia Brasiliana em diferentes graus metamórficos, foram investigadas neste trabalho. Quatro tipos de rochas contendo granada foram selecionados: granulito de ultra-alta temperatura, granito granulitizado, paragranulito e cianita-granadamuscovita xisto. Proporções moleculares indicam variações significativas nos conteúdos de $\mathrm{Fe}$ e $\mathrm{Mg}$ e pequenas variações do $\mathrm{Ca}$ e $\mathrm{Mn}$. Todos cristais de granada analisados pertencem à série almandinapiropo. Granada do mica xisto apresenta maior quantidade (\%) de moléculas de $\mathrm{Fe}$ $\left(\mathbf{a l m}_{76} \mathbf{p y} \mathbf{y}_{15}\right.$ gro $\left._{6} \mathbf{s p}_{2}\right)$ seguidas por granada de granito granulitizado ( $\left.\mathbf{a l m} \mathbf{6 9 - 6 6}_{\mathbf{2 0 - 2 6}} \mathbf{p y}_{\mathbf{2 0}} \mathbf{g r o}_{6-9} \mathbf{s p}_{2-3}\right)$, paragranulito $\left(\mathbf{a l m}_{62-52} \mathbf{p y}_{42-32} \mathbf{g r o}_{4-6} \mathbf{s p}_{1-2}\right)$ e granulito de ultra-alta temperatura $\left(\mathbf{a l m}_{63} \mathbf{p y}_{23} \operatorname{gro}_{13} \mathbf{s p}_{1}\right)$.

$\mathrm{Em}$ todos cristais as bordas de granada possuem maior conteúdo de $\mathrm{Fe}$ que os núcleos. $\mathrm{Mg}$ e $\mathrm{Ca}$ possuem relação inversa ao $\mathrm{Fe}$ em granada do granulito de ultra-alta temperatura, mostrando empobrecimento daqueles elementos em direção às bordas do cristal. Cristais de granada do granulito de ultra-alta temperatura e de granito granulitizado possuem idades isocrônicas Sm-Nd mais antigas (625$633 \mathrm{Ma})$ e maiores valores de $\mathrm{Mg} /(\mathrm{Mg}+\mathrm{Fe})$. Granada de paragranulito possui idade isocrônica $\mathrm{Sm}-\mathrm{Nd}$ de $606 \mathrm{Ma}$ e maiores valores de $\mathrm{Ca} /(\mathrm{Ca}+\mathrm{Mg})$. A dispersão parece indicar que o sistema isotópico da granada de paragranulitos permaneceu aberto por mais tempo devido a reações retrometamórficas ou evento deformacional.

\section{INTRODUÇÃO}

Os minerais do grupo da granada (grupo espacial cúbico $I a 3 d$ e $Z=8$ ) são soluções sólidas entre dois ou mais membros finais, cuja fórmula estrutural pode ser descrita por $\mathrm{X}_{3}{ }^{[8]} \mathrm{Y}_{2}{ }^{[6]}\left[\mathrm{SiO}_{4}\right]_{3}$, onde $\mathrm{X}=\mathrm{Mg}^{2+}, \mathrm{Fe}^{2+}$, $\mathrm{Mn}^{2+}$ ou $\mathrm{Ca}^{2+}$ e $\mathrm{Y}=\mathrm{Al}^{3+}, \mathrm{Fe}^{3+}, \mathrm{Ti}^{4+}, \mathrm{Cr}^{3+}$ ou V ${ }^{3+}($ Deer et al. 1982). Embora encontrados em rochas ígneas, os minerais das várias séries isomórficas do grupo da granada são mais comuns em rochas metamórficas, podendo estar presentes em diferentes litotipos, sob diferentes condições de pressão e temperatura. Essas características tornam esses minerais excelentes indicadores petrogenéticos, principalmente quando estudados por meio de diferentes técnicas analíticas.

Neste contexto, padrões geoquímicos de granada dentre outros minerais têm sido objeto de pesquisa nas últimas décadas com a finalidade de obter informações sobre processos geológicos (Mezger et al. 1988,1989; Burton et al. 1995). Um deles é o comportamento do sistema isotópico de minerais portadores de elementos terras raras e isótopos radiogênicos em que a granada tem sido amplamente utilizada (Mezger et al. 1991, 1992; Harrison \& Wood 1983, Eeckhout et al. 2002).

$\left({ }^{*}\right)$ Centro de Pesquisa Manuel Teixeira da Costa, ICG/UFMG, Belo Horizonte. E-mail: dpiuzana@yahoo.com.br

$\left(^{* *}\right)$ Laboratório de Geocronologia, Instituto de Geociências, UnB, Brasília. $\left.{ }^{* * *}\right)$ Laboratório de Microanálises, UFMG/CDTN, Belo Horizonte. 